\title{
Targeting AVIL, a New Cytoskeleton Regulator in Glioblastoma
}

\author{
Robert Cornelison ${ }^{1,+}+\mathbb{C}$, Laine Marrah ${ }^{1,+}+\mathbb{D}$, Drew Horter ${ }^{1,+}{ }^{\text {, Sarah }}$ Lynch $^{1}$ and Hui Li ${ }^{1,2, *}$ \\ 1 Department of Pathology, School of Medicine, University of Virginia, Charlottesville, VA 22908, USA; \\ jrc3hg@virginia.edu (R.C.); lm2ku@virginia.edu (L.M.); dah6zq@virginia.edu (D.H.); sl7hx@virginia.edu (S.L.) \\ 2 Department of Biochemistry and Molecular Genetics, School of Medicine, University of Virginia, \\ Charlottesville, VA 22908, USA \\ * Correspondence: h19r@virginia.edu; Tel.: +1-434-982-6624 \\ + These authors contributed equally.
}

Citation: Cornelison, R.; Marrah, L.; Horter, D.; Lynch, S.; Li, H. Targeting AVIL, a New Cytoskeleton Regulator in Glioblastoma. Int. J. Mol. Sci. 2021 22, 13635. https://doi.org/10.3390/ ijms222413635

Academic Editors: Jaroslaw

Maciaczyk, Hugo Guerrero-Cazares and Amit Sharma

Received: 23 November 2021 Accepted: 16 December 2021 Published: 20 December 2021

Publisher's Note: MDPI stays neutral with regard to jurisdictional claims in published maps and institutional affiliations.

Copyright: (c) 2021 by the authors. Licensee MDPI, Basel, Switzerland. This article is an open access article distributed under the terms and conditions of the Creative Commons Attribution (CC BY) license (https:// creativecommons.org/licenses/by/ $4.0 /)$.

\begin{abstract}
Glioblastoma (GBM) is the most common adult neural malignancy and the deadliest. The standard of care is optimal, safe, cytoreductive surgery followed by combined radiation therapy and alkylating chemotherapy with temozolomide. Recurrence is common and therapeutic options in the recurrent setting are limited. The dismal prognosis of GBM has led to novel treatments being a serious roadblock in the field, with most new treatments failing to show efficacy. Targeted therapies have shown some success in many cancers, but GBM remains one of the most difficult to treat, especially in recurrence. New chemotherapeutic directions need to be explored, possibly expanding the targeted chemotherapy spectrum in previously unforeseen ways. In this perspective paper, we will explain why AVIL, an actin-binding protein recently found to be overexpressed in GBM and a driving force for GBM, could prove versatile in the fight against cancer. By looking at AVIL and its potential to regulate FOXM1 and LIN28B, we will be able to highlight a way to improve outcomes for GBM patients who normally have very little hope.
\end{abstract}

Keywords: glioblastoma; AVIL; FOXM1; LIN28B; actin; gelsolin

\section{Introduction}

Glioblastoma multiforme (GBM) is the grade IV astrocytoma. As one of the most common and malignant adult neural malignancy, it affects approximately 14,000 people in the United States per year [1-4]. Astrocytomas are malignancies arising from astrocytes divided into four grades: pilocytic astrocytoma (grade 1, generally non-invasive, pediatric masses), astrocytoma (grade II), anaplastic astrocytoma (grade III), and GBM (grade IV). GBMs are further divided into primary and secondary subtypes based on both biology and differences in overall molecular evolution. The current standard of care is cytoreductive surgery followed by the combination of radiation therapy and alkylating chemotherapy with temozolomide [3-7]. Primary GBMs are thought to arise de novo as the grade IV lesion, and are generally associated with an aging population (age > 50), EGFR amplification, loss of heterozygosity $(\mathrm{LOH})$ at 10q, p16 deletion, and mutation of PTEN [8]. Secondary GBMs evolve from lower grade II-III lesions over several years and are seen in younger patients [9]. Overall life expectancy for primary GBM is only 12-15 months, while lower grade III anaplastic astrocytoma patients survive for 2-5 years [1]. GBM prognosis is so dismal that long-term survivors (LTS) are clinically defined as living $>36$ months after diagnosis with only $2-5 \%$ meeting this criteria $[10,11]$. The hallmark GBM histologic finding is microvascular proliferation and areas of pseudopalisading necrosis [12,13]. World Health Organization (WHO) classification now separates GBMs into two subgroups by isocitrate dehydrogenase (IDH) gene mutation status, with wild-type IDH accounting for $90 \%$ of cases seen $[2,14,15]$. Molecular categorization clusters GBMs into four subgroups [16], as seen in (Figure 1). 


\section{Grade I: Pilocytic}

Non-invasive, pediatric, cystic consistency

Survival: $>10$ years

\section{Grade II: Astrocytoma}

Typically in adults, may progress to secondary glioblastoma

Survival: $>5$ years

\section{Grade III: Anaplastic astrocytoma}

Typically found in adults, may progress to secondary glioblastoma

Survival: $2-5$ years

\section{Grade IV: Glioblastoma multiforme}

Most common malignant brain tumor, poor prognosis

Survival: 1 year

Primary: evolves de novo, older patients, EFGR amplication, LOH at 10q; 16 deletion/mutation of PTEN11

Secondary: evolves from Grade II-III, evolves over years, younger patients

$\rightarrow$ Neural: expression of neuron markers including NEFL, GABRA1, SYT1, and SLC12A5

Proneural: PDGFRA alterations, IDH1 point mutations, lack of chromosome 7

$\longrightarrow$ amplification paired with chromosome 10 loss

Classical: chromosome 7 amplification paried with chromosome 10 loss, EFGR amplification, lack of

TP53 mutation, CDKN2A deletion

Mesenchymal:NF1 deletion, increased activity of mesenchymal (CHI3L1 and MET) and astrocytic

markers (CD44 and MERTK), increased expression of tumor necrosis pathway

\section{New WHO Guidelines 2021:}

IDH mutant glioblastoma > Astrocytoma grade 4

IDH mutant $+1 \mathrm{p} / 19 \mathrm{q}$-codeletion > Oligodendroglioma grade 2,3

Glioblastoma > IDH wildtype with common alterations: HTERT promoter mutation, EGFR amplification, loss of chromosome 10 and gain of chromosome 7

Figure 1. Overall classification of the common forms of astrocytomas. Guidelines from the World Health Organization were updated in 2021. The overall heterogeneity of CNS tumors makes nomenclature and histologic distinction a critical but challenging task in the wake of novel technologies [17,18]. Treatment options at presentation have remained relatively stable for many years with few novel treatments or targeted therapies showing efficacy. The current standard of care is surgery followed by combination radiotherapy and temozolomide chemotherapy in the case of glioblastoma with methylated $\mathrm{O}^{6}$-methylguanine-DNA-methyltransferase (MGMT). In cases with unmethylated MGMT, the addition of alternating electric fields therapy has shown promise in clinical trials and is included in front-line treatment in the National Comprehensive Cancer Network (NCCN) guidelines. Due to its tendency to be hypervascularized, the anti-angiogenic bevacizumab is commonly used as a second-line therapeutic in combination with agents like the alkylating mustard carmustine or lomustine [19-21]. Resistance to these therapies is based on two major factors: maintenance of stemness and overall dissemination. GBMs rarely metastasize to distant sites, but, through an EMT-like process (glial-to-mesenchymal transition) specific to the brain, they can disseminate widely throughout the CNS, making GBM a disease of the entire brain [22-24].

Cytotoxic chemotherapy has long been a successful, yet problematic treatment for many types of cancer. With each class of chemotherapeutics comes a diverse list of toxicities that make it difficult to distinguish between adverse effects and disease pathology [25]. Antimitotic agents have proven to be very effective in the killing of cancer cells, but by acting on mitosis, lend themselves to causing additional cell death throughout the body. The move to cancer-specific, targeted therapies began with BCR-ABL fusion targeting using imatinib [26]. This opened the cancer field to an entirely new philosophy of therapeutic options in identifying and characterizing differentially expressed genes in cancer as lead targets for pharmaceutical intervention. The initial discoveries of cancer-specific proteins to inhibit led to drugs targeting MEK, b-RAF, ALK, and other major signaling network controllers. However, only $5-7 \%$ of novel targeted therapies demonstrate significant efficacy and make it through clinical trials in solid tumors. The overall tumor heterogeneity combined with the complex, redundant signaling networks seen in most solid tumors makes specific, gene-level inhibition unlikely to defeat most advanced cancers. 
In reality, the promise of ultra-specific, targeted therapies has yet to be fully realized, especially in aggressive malignancies like GBM. Solid tumors are rapidly evolving entities, and adding specific selection pressures in the form of targeted inhibitors to genetically unstable, rapidly dividing tumors leads to a predictable outcome: chemoresistant, untreatable cancer cells. This has led the targeted therapy field to step back from ultra-specific monotherapies and look for more broad range inhibitors that target entire families of kinases. Imatinib, while being designed to target the cancer-specific BCR-ABL fusion, has been shown to also target other kinases in cancer [22], making it a promising treatment for use outside of cancers with the Philadelphia chromosome. This underscores the importance of moving away from monotherapies and using well-thought-out, outcome-directed combination therapies meant to enhance specific targeting of a pathway or block therapy bypass mechanisms.

Combination therapies are critical for targeted inhibitor success in many cases, as using dual inhibition of the same pathway can lead to synergistic effects. Inhibiting activating mutations of RAF kinases has shown promise in susceptible melanoma patients, but combining RAF inhibition with MEK inhibitors can lead to better outcomes [27-30]. These combined treatments unfortunately also lead to severe side effects and, in many cases, toxicities requiring termination of treatment. Instead of targeting specific families of enzymes or other "druggable" proteins, there has always been the prospect of identifying cancer-specific genes that mimic the targets of the original cytotoxic chemotherapies, such as tubulins and other cytoskeletal proteins. In this way, they retain their increased specificity to cancer, but by targeting critical cell infrastructure can induce widespread damage to multiple intracellular systems simultaneously.

The idea of identifying a cancer-specific cytoskeletal protein is not new. Some of our strongest cytotoxic agents target the cytoskeleton and inhibit mitosis. The action being primarily on dividing cells gives some cancer specificity in that at least they target rapidly dividing cells. The downside of the approach has been simply that the body has many rapidly dividing cell types, leading to many deleterious toxicities. Chemotherapies targeting the cytoskeleton began in the 1960s with the use of vincristine, a vinca alkaloid, targeting tubulin and disrupting polymerization. Targeting tubulin as a means of preferentially attacking rapidly dividing malignant cells has dominated cytoskeletal agents with the most commonly used being the vinca alkaloids, the taxane stabilizing family (paclitaxel and docetaxel), and the newer epothilones [31-33]. Epothilones have come to the forefront of research by demonstrating less toxicity and more specificity, but overall side effects remain high in cytoskeletal targeting. Importantly, disrupting tubulin specifically is catastrophic to all dividing cells, whether malignant or not. A differentially expressed, non-tubulin cytoskeletal protein that could be targeted with a reasonable therapeutic index has been a difficult find. Actin, on the other hand, is required for many malignant phenotypes including metastatic spread, cytokinesis, and cell motility. Recently, our lab discovered a previously seen but relatively uncharacterized oncogenic actin-binding protein, advillin (AVIL), to be upregulated in GBMs as well as other cancers [34]. It is rarely expressed outside of embryonic stages, and in GBM appears to be critical for maintaining tumorigenicity. Advillin may be a relatively cancer-specific cytoskeletal protein that sits at the crossroads of the two therapeutic philosophies: broad range cytotoxic agents that target machinery required for division and a cancer-specific targeted therapy that limits toxicities in normal cells.

In this manuscript we will give a brief overview of AVIL and its functions, a summary of the known family members and their proto-oncogenic roles, roles of downstream targets of AVIL and their specific functions in GBM, and look at AVIL functions specifically involved in GBM dissemination and immune system evasion. Finally, we will speculate the therapeutic potential of targeting AVIL in GBM. 


\section{Advillin Overview}

Advillin, encoded by the AVIL gene, is one of the members of the gelsolin superfamily of calcium-dependent, actin-binding proteins $[35,36]$. Actin-binding proteins remodel actin in several different ways and act as modifying components of the overall cytoskeletal network. Advillin can nucleate filaments and promote polymerization, bundle actin to create cytoskeletal networks, disassemble actin through severing and depolymerization, and cap actin at either the pointed or barbed end [36,37]. The role of actin-binding proteins in cancer has been under substantial investigation. The actin cytoskeleton is responsible for a broad range of cellular phenotypes such as maintenance of polarity, movement, invasion, endocytosis, cell division, and trafficking [38]. For cancers to proliferate and invade, the actin-binding proteins are essential for these functions by how they polymerize, depolymerize, and bundle actin using intracellular calcium, and a host of chaperone proteins depend upon the localization and essential function. Advillin appears to be a critical actin-binding protein involved in bundling actin in response to stress and movement [39].

Advillin was identified in an adult murine brain cDNA screen to share $65-75 \%$ homology to villin, gelsolin, and adseverin [35]. Similar to other gelsolin superfamily members, Advillin contains six domain structures, termed gelsolin-like (G)1-6, with a carboxyterminus headpiece domain [40], as shown in (Figure 2). The G1 and G4 domains have been found to bind actin monomers, while G2 binds filaments. The headpiece also contains F-actin binding activity, which has been shown to be involved in actin bundling [41]. Gelsolin contains the G1-6 but does not have the headpiece domain. The headpiece domain seen in advillin is shared with villin (VIL1), villin-like (VILL), flightless 1, and supervillin [40].

Advillin structure
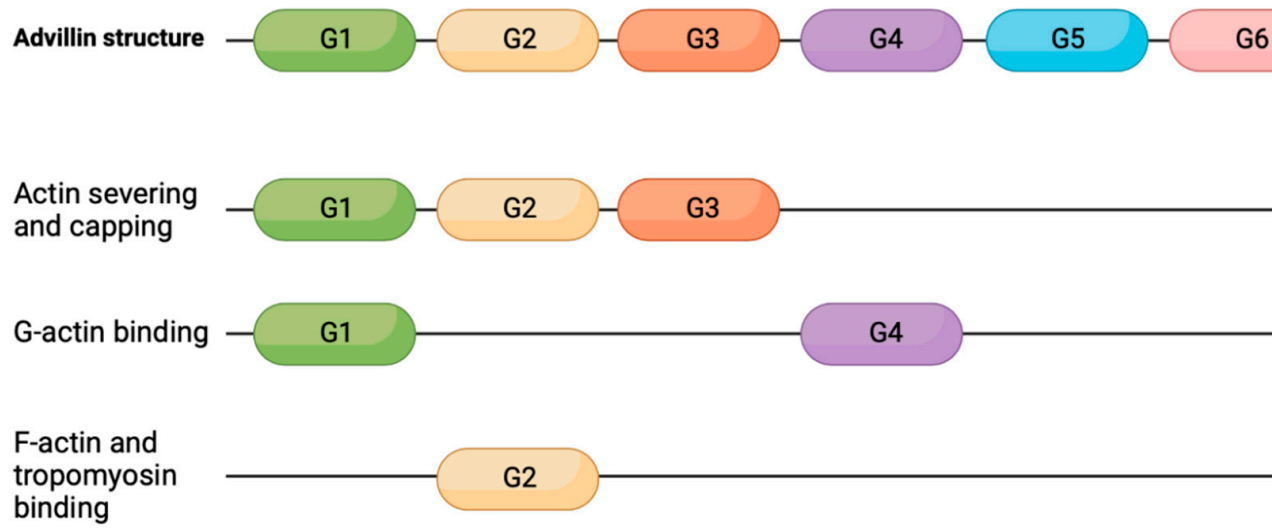
F-actin and
tropomyosin
binding

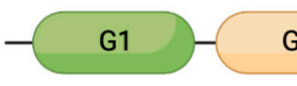

G2 G3

G1 G4

\section{G2}

Actin bundling

$\mathrm{PIP}_{2}$ binding

G1 G2

Inhibition of G1-3 function in absence of $\mathrm{Ca}^{+}$

Figure 2. Advillin protein structure and domain functions.

AVIL is heavily overexpressed in almost $100 \%$ of GBMs, while the gene products are virtually non-existent in normal brain tissue [34]. This overexpression was also confirmed via immunohistochemistry assays that were performed on normal brain and glioblastoma tissues. The gene is essential for the proliferation and migration of GBMs [34]. The siRNAmediated silencing of AVIL resulted in a reduction of cell motility measured by wound healing and live cell imaging, and eventually killed cancer cells expressing AVIL, while 
the same siRNAs on astrocytes had no obvious effect. In contrast, overexpressing AVIL enhanced cell proliferation and migration and transformed astrocytes in vivo. Clinically, higher AVIL expression is associated with worse patient outcomes in GBM as well as lower grade gliomas [34]. A summary of evidence supporting AVIL as a lead target in GBM is shown in Table 1.

Table 1. Overview of AVIL as a lead target in GBM.

\begin{tabular}{c} 
Advillin (AVIL) as a Lead Target in GBM \\
\hline $\begin{array}{c}\text { Expression: AVIL is overexpressed in 100\% GBM cell lines and clinical samples, but hardly } \\
\text { expressed in astrocyte and normal brain tissues. }\end{array}$ \\
$\begin{array}{c}\text { Loss-of-function systems: Silencing AVIL caused reduced proliferation and migration of GBM cell } \\
\text { culture and xenograft, but had no effect on astrocytes. }\end{array}$ \\
Gain-of-function systems: Overexpressing AVIL promoted GBM and astrocyte cell proliferation \\
and migration, and transformed astrocytes. \\
\hline GBM stem cell/initiating cells: The major therapy resistant cells. GSC/GIC cells express even \\
higher levels of AVIL. Silencing AVIL triggered reduced neurosphere formation and stemness. \\
Clinical correlation: High AVIL expression is correlated with worse patient survival. \\
\hline
\end{tabular}

While the exact regulation mechanisms of the advillin are still under investigation, it and similar proteins from its family could be intricately involved in the formation of our most deadly cancers. With its versatile function, it could be hypothesized that AVIL can carry out the functions of other gelsolin/villin family proteins in tumor cells.

\section{Advillin Family Members and Their Association with Cancer}

With important functions in cell motility, ECM interactions, and stress responses, it is unsurprising that gelsolin/villin superfamily members have been found to play roles in tumorigenesis. Gelsolin has been found to regulate EMT in HPV cells, predict patient prognosis in squamous cell carcinoma of the larynx, and suppress apoptotic machinery in neuroendocrine prostate cancer [42,43]. Adseverin expression has been reported to be a marker for cisplatin resistance in bladder cancer and correlate with prognosis in colorectal cancer [44,45]. Calcium signaling is disrupted in GBM, and may provide an abundant source for gelsolin family member activation [46]. PIP2 negatively regulates gelsolin family members, and it is well known that PI3K upregulation in cancer phosphorylates PIP2 to PIP3. This dysregulated signaling may lead to disrupted advillin function in cancer, allowing for promotion and metastasis of GBM.

Glioblastoma is characterized by both resistance to apoptosis and an invasive phenotype. In normal cells, the balance between the pro- and anti-apoptotic machinery regulates mitochondrial membrane permeability, with loss of integrity leading to mitochondrialdependent cell death. Cellular insults mediated through DNA damage, hypoxia, reactive oxygen species, and nutrient deprivation push this balance to a threshold that results in release of APAF-1, AIF, and cytochrome c from the mitochondria into the cytosol, activating the classical apoptosomes, leading to caspase-dependent death. In cancer, the anti-apoptotic signals raise the threshold of stress and damage required to die and lead to survival of malignant cells. Gelsolin has been found to inhibit apoptosis through multiple mechanisms, including direct interactions with the mitochondria. It interacts with mitochondrial gatekeeper VDAC1, stabilizing the mitochondria and preventing loss of mitochondrial membrane integrity [47]. In this way it can act as common proto-oncogenic BH3 family of anti-apoptotic proteins raising the threshold required for cell death to occur. Glioblastomas also have constitutive caspase- 3 activity that promotes cell motility without inducing apoptosis [48]. Gelsolin is an established substrate of caspase-3, producing a cleaved c-terminal fragment that was shown to promote invasion, migration, and metastasis of melanoma cells. Further, the caspase- 3 cleaved-gelsolin fragment shows higher efficiency at cleaving F-actin, influencing migration potential [48]. For AVIL specifically, after intracranial xenografts in tumors where AVIL was knocked out by shRNA, there were significantly more apoptotic cells than in the control group [34]. Adseverin, an actin 
capping and severing protein of the gelsolin family, has also been found to protect against apoptosis-mediated cell death [49]. This indicates that loss of AVIL function inhibits the tumor's ability to escape some tumor-suppressing mechanisms via apoptosis.

It has also been shown that gelsolin is key in the inhibition of angiogenesis. To grow and metastasize, tumor cells need a large number of sustained nutrients, oxygen, and the ability to dispose of waste [46]. They can obtain this by inducing the formation of new blood vessels that feed directly to the tumor (angiogenesis), and it has been shown that, in the absence of angiogenesis, the generation of lethal tumor mass is not possible [47]. Endothelial cells with gelsolin knocked out showed significantly higher levels of migration, and when gelsolin was overexpressed, the inhibition of angiogenesis was restored [48]. Furthermore, vascular endothelial growth factor (VEGF) is the primary signal that recruits endothelial cells to migrate [49], and the actin cytoskeleton of endothelial cells is inherently involved in the response to VEGF, which dictates the route for the vascularization of the tumor. AVIL, on the other hand, induces larger, more deadly tumors when overexpressed [45], and thus, angiogenesis is most likely induced by extensive nucleation and growth of actin filaments facilitated by AVIL.

Gelsolin has also been found to be packaged into exosomes and secreted, termed plasma gelsolin (pGSN), with this form inhibiting CD8+-mediated tumor immune surveillance [50-52]. In this context, secreted gelsolin has been shown to inhibit neoantigen presentation to conventional dendritic cells in the tumor microenvironment, leading to immune evasion with a subsequent increase in glutathione (GSH) expression. Increases in GSH in ovarian cancer increase resistance to platinum chemotherapy [51]. The implication that autocrine signaling through exosomal gelsolin secretion leads to multiple oncogenic phenotypes underscores the breadth of functional roles the gelsolin superfamily of actinbinding proteins can play. A summary of the characterized gelsolin family members' functional roles on actin is seen in (Figure 3).

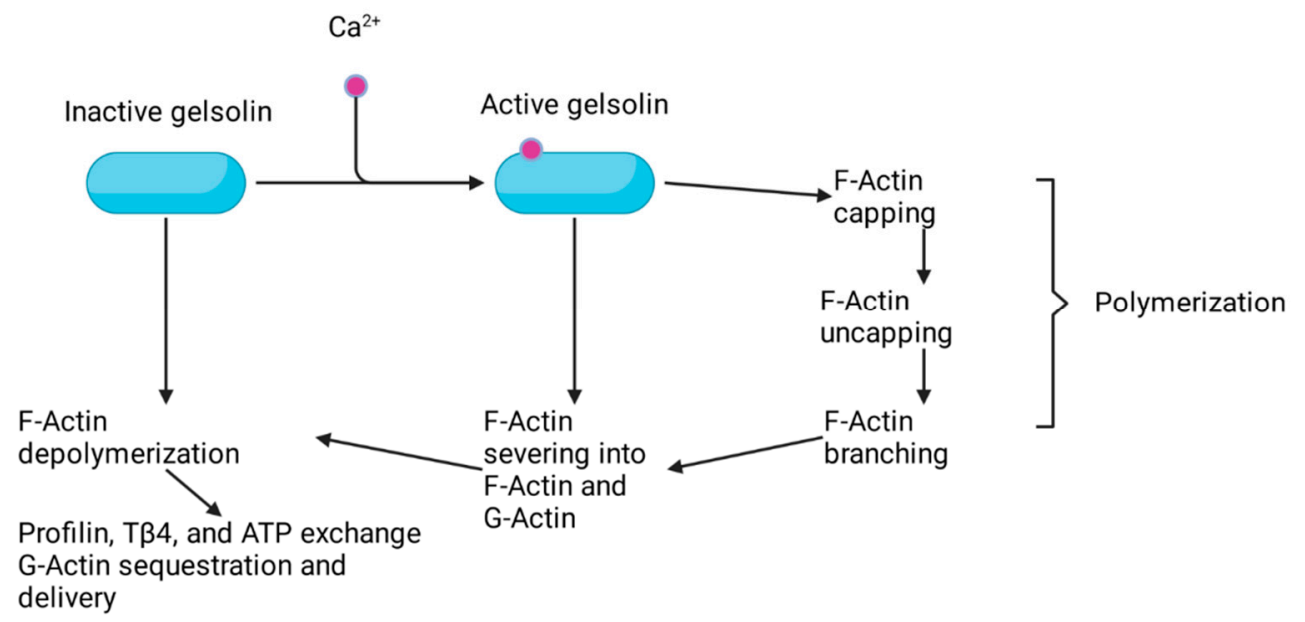

Figure 3. Overview of gelsolin activation through calcium binding and roles in modulating actin structural changes.

Another gelsolin family member, macrophage-capping protein (CapG), is proposed to be a diagnostic biomarker, indicator of prognosis, and predictor of response to treatment across numerous cancer types [53]. High levels of CapG expression have been found to correlate with lymph node metastasis and advanced TNM stage in various cancer types, including glioma [10,53-55]. CapG caps the barbed end of actin and inhibits polymerization. Notably, though, growing evidence has supported CapG having non-canonical tumorpromoting activity. In glioma cell lines U251 and U87, overexpression of CapG promoted proliferation while CapG knock-down inhibited cell cycle progression and resulted in an increase of G0/G1 cell cycle arrest and a decrease in cells in G phase [55]. CapG expression also promoted cell migration and invasiveness in glioma cell lines [55]. CapG localizes to both the cytoplasm and nucleus, where it plays a role in invasive phenotypes. 
Nuclear CapG was found to bind to the epigenetic regulator arginine methyltransferase PRMT5 [56]. PRMT5 methylates histones and is an epigenetic repressor, and PRMT5 inhibition has antitumor effects in glioblastoma models. Gelsolin has been found to be upregulated following IL-6 induction of neuroendocrine transdifferentiation in prostate cancer cells [43] and also to be a coactivator for nuclear receptors including STAT3 and androgen receptor $[43,57]$.

\section{Downstream Targets of AVIL: FOXM1}

FOXM1 is a transcription factor belonging to the fox family of proteins unified by its conserved DNA-binding domain known as the forkhead box [58]. Despite a common domain, Fox proteins exhibit a wide range of regulatory activity [59]. FOXM1 regulates several cyclins and kinases and is key to appropriate progression through the cell cycle and mitosis, and the cells of mice without the Foxm1 gene fail to enter mitosis [59]. This transcription factor is mostly expressed in fetal tissue [60] and is typically not expressed in fully differentiated cells [58]. The regulation of FOXM1 is achieved predominantly through phosphorylation, which increases transcriptional activity, but post-transcriptional modification is involved as well [59]. Furthermore, its upregulation has been found to correlate to a variety of cancers including GBM [58]; as such, increased FOXM1 activity could promote cell cycle progression beyond what is appropriate and lead to the formation of tumors [59].

The FOXM1 gene contains nine exons and ultimately translates into two alternatively spliced isoforms called FOXM1A and FOXM1B [60]. FOXM1B is the only isoform found to be expressed in glioma cells, implying that this is the isoform relevant to gliomas [60]. FOXM1B, when transfected into mice, caused them to develop aggressive tumors. Inhibiting FOXM1B expression in other mice via siRNA led to no brain tumors and significantly increased survival, implying that FOXM1B has tumorigenic properties [60].

Additionally, a correlation has been established between FOXM1 levels and the grade of astrocytoma, with GBM consistently exhibiting the highest levels of the transcription factor while normal brain tissue does not express it $[58,60]$. An absence of FOXM1 correlates to significantly longer survival in GBM patients, all of which indicates a potential connection between FOXM1 and tumor progression [58]. FOXM1 binds to the promoter of VEGF, the primary growth factor involved in angiogenesis that is frequently overexpressed in glioma cell lines [58]. The absence of FOXM1 corresponds to lowered VEGF in vitro, and, in mouse experiments, glioma cells with FOXM1 inhibited were found to have lower VEGF than their counterparts [58]. As such, the interaction between FOXM1 and VEGF is key in driving angiogenesis in glioma cells, which is an important factor in tumorigenesis.

FOXM1 has been linked to EMT in various tumors. It activates MMP-2, which is an enzyme critical to breaking down the extracellular matrix and allowing tumor penetration into the brain. Moreover, MMP-2 is involved in mesenchymal phenotypes in GBM, and increased MMP-2 directly correlates to increased EMT [58]. FOXM1 is, therefore, implicated in the process of tumor invasion, which is a hallmark of GBM as well [58].

When AVIL expression is altered, many of the genes with inversely affected expression are targets of FOXM1. More directly, AVIL affects levels of FOXM1 protein but does not affect levels of its mRNA, implying a post-transcriptional regulation [34]. Additionally, there is a direct correlation to half-life, where silencing AVIL reduces FOXM1 half-life and overexpressing AVIL extends FOXM1 half-life [34]. It is postulated that this could be because disrupting F-actin dynamics via silencing AVIL decreases FOXM1 stability [34]. As a result, it has been suggested that FOXM1 is downstream of AVIL [34], and the interaction between the two has potential for elucidating the link between AVIL and tumorigenesis.

\section{FOXM1 Regulation of LIN28B and Its Role in GBM}

LIN28 is an RNA-binding protein associated with oncogenic phenotypes. Higher LIN28 expression has been found to correlate with poorer survival in GBM patients [61]. LIN28 is known to interact with the let-7 family of microRNAs that typically function 
to suppress tumors, but often have reduced expression in cancer cells [62]. There are two homologs of LIN28, LIN28A and LIN28B, both of which can be expressed in glioma cells [63]. Notably, silencing either AVIL or FOXM1 reduces LIN28B expression; likewise, overexpressing AVIL or FOXM1 increases LIN28B expression. Additionally, expressing LIN28B in cells with silenced AVIL led to some rescuing of tumorigenic effects including proliferation and migration. AVIL has also been found to inversely correlate with let-7 levels [34]. This indicates that LIN28B is likely downstream of both AVIL and FOXM1 [34]. The hypothesized pro-tumor roles of AVIL specifically through the FOXM1/LIN28B axis is seen in (Figure 4).

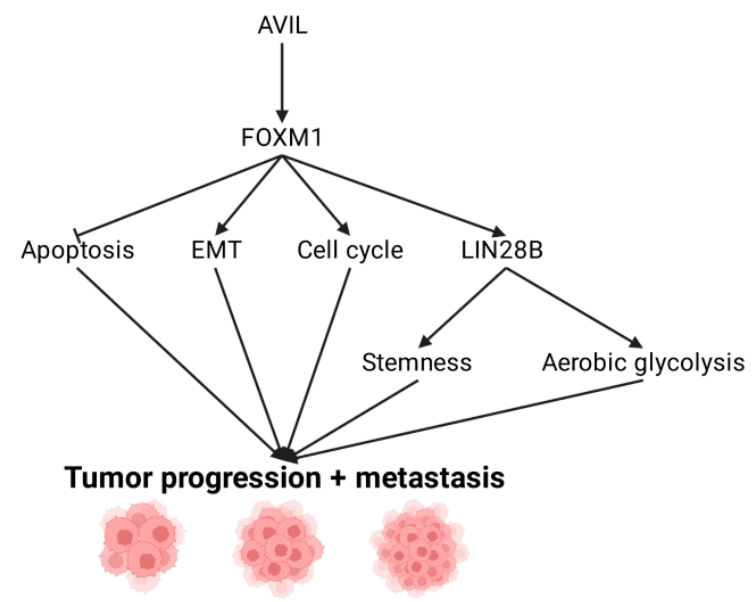

Figure 4. Overview of AVIL oncogenic functions through the FOXM1-LIN28 axis.

LIN28B is overexpressed in cancer cell lines and its overexpression correlates with decreased let-7 and therefore, increased expression of let-7's oncogenic targets [62]. This occurs because LIN28B blocks let-7 precursors from processing to become mature RNA, therefore inhibiting the production of let-7 miRNAs [62]. Furthermore, LIN28 may play a role in cell cycle progression in glioma cells, which could lead to increased cell proliferation [61]. Overall, it is most likely involved in oncogenic pathways via its interactions with let-7 miRNAs, AVIL, and FOXM1.

Another connection between FOXM1 and LIN28 is that FOXM1 also regulates LINC01094, which acts as a miRNA sponge for MIR-577, a well-characterized tumor suppressor that controls LIN28, as well as b-catenin/wnt signaling and EMT [64,65]. The LINC01094/MIR577 axis has been shown to control pancreatic cancer progression and metastasis through activation of AKT [64]. It has also been shown to confer radioresistance in renal cell carcinoma specifically through a feedback loop including CHEK2 and FOXM1 [65].

\section{Activating Invasion and Metastasis through EMT}

In order to migrate and metastasize, cancer cells take advantage of the epithelialmesenchymal transition, also called the glial-to-mesenchymal transition (EMT), which is a normal cellular event. During this transition, the cells change their cell-to-cell contacts and are able to move through the extracellular matrix to invade new tissues as a group [66]. It appears that the actin cytoskeleton is heavily involved in the entire transition [67], but its influence is most easily seen in the formation of filipodium and invadopodium. These organelles are vital for the intrusion into new tissues and drive the movement of the tumor cells. The actin-rich structures degrade the extracellular matrix, which allows the tumor to enter new tissues and/or the bloodstream [67].

EMT-like phenotypes in progressing tumor cells have been well documented [68]. In the case of GBM, it is important to make the distinction between EMT and EMT-like phenotypes because EMT indicates that the tumor is epithelial in origin, which is not true of GBM [68]. Our lab found that when silencing AVIL in A172 GBM cells (IDH wild-type/P53 wildtype), there was a dramatic reduction in cell movement in a wound-healing assay [34]. 
This result was confirmed with live-cell imaging after AVIL knockout. This could indicate that the loss of AVIL function can interrupt the tumor's ability to invade neighboring brain tissue. Furthermore, AVIL and other villin family proteins have shown to be closely related and colocalized to Arp 3. Additionally, in kidney podocytes, AVIL was found to be integrated with PLCE1, F-actin, and the ARP complex, especially in lamellipodia [39]. Interference with this complex could inhibit the formation of lamellipodia and limit cancer's ability to spread.

\section{Escaping Immune Surveillance}

Natural killer (NK) cells are part of the innate immune system and can coordinate the spontaneous killing of tumor cells [69]. In breast cancer, the involvement of the actin cytoskeleton plays a role in the resistance of tumor cells against NK cells. After NK cells were introduced to a breast adenocarcinoma cell line, there was a large buildup of actin at the contact point that protected the cells from NK-mediated lysis [70]. Furthermore, the inhibition of the Arp 2/3 complex reduced the ability of the tumor cells to form the rapid actin remodeling and made the tumor cells more susceptible to NK lysis by $50-150 \%$ [70]. Because AVIL is involved in the formation of dynamic structures such as lamellipodia and filopodia, it is logical to think that AVIL could also be involved with the Arp 2/3 actin assembly complex in the rapid actin response to NK cells. This shows yet another way that an AVIL-based treatment could be effective.

\section{Discussion}

Glioblastoma remains one of the most difficult malignancies to treat with few recent advances seen as truly changing outcomes. The overall complexity of dealing with a primary malignancy of the brain combined with the insidious spread of tumor-initiating cells away from the main lesion makes the development of novel therapeutics a major roadblock in the field. While AVIL may appear superficially to be just another targeted therapy, its role as a major cytoskeletal protein may hold the key to a new generation of targeted therapies that disrupt critical cell infrastructure. Targeting a differentially expressed, cytoskeletal protein may hold promise in keeping toxicity to a minimum while still achieving inhibition across many systems at once. AVIL's overexpression in tumor cells and lack of expression in noncancerous tissue is promising by itself [34]. It becomes an even stronger candidate when discussing its involvement in known cancer pathways and mechanisms as well as that of the gelsolin superfamily. AVIL's close relation to the protein gelsolin gives a mechanism by which its knockout could be a death sentence for GBM cells.

Downstream of AVIL, FOXM1 is implicated in angiogenesis, tumor invasion, and EMT [58], while LIN28B allows expression of oncogenes by inhibiting let-7 miRNAs [62]. The evidence of AVIL acting upstream of both FOXM1 and LIN28B indicates that it likely plays a role in tumorigenesis and proliferation [34]. Additionally, AVIL's direct role in actin filament modulation provides other mechanisms by which a treatment can act to kill GBM cells [39]. Actin affects tumor mobility, metastasis, and invasion, and treatments targeting AVIL would be able to target the cytoskeleton without affecting tubulin, thus avoiding some deleterious effects in noncancerous dividing cells throughout the body. The prospect of targeting any critical cellular infrastructure can lead to deleterious toxicities regardless of the initial targeting specificity. Pharmaceutical inhibition of actin-binding proteins may be a difficult undertaking in terms of small molecule inhibitors due to the lack of a traditional active site. The gelsolin family members are also quite conserved and care will have to be taken to target AVIL specifically without inhibiting other critical family members. Targeting the headpiece domain or other regions relatively specific to AVIL will be critical in drug design decisions moving forward. Downstream family members could also make specific targeting of the system a reality, but the potential of utilizing AVIL as a means of hitting the entire cytoskeletal system only in cells actively expressing AVIL protein may be the most advantageous considering the malignant potential of GBM cells overall. Modulation of AVIL protein in the cell may also require multiple inhibitors as specific roles of AVIL are 
more thoroughly elucidated. Rational drug design from atomic-scale starting points may also be required as actin-binding proteins are not commonly included in druggable protein collections. Novel assays for AVIL-specific, oncogenic functional roles in growing tumors will be critical in identifying optimal pharmacologic inhibitors as the field progresses.

As previously mentioned, the metastatic process specific to glioblastoma in terms of cell fate transition is a complex mix of epithelial and mesenchymal gene-specific changes termed glial-to-mesenchymal transition. The players involved include well-known members such as SNAIL and E- and N-cadherin but the interplay between these genes and actin cytoskeletal changes induced by aberrant expression of AVIL still require more research to fully elucidate [71]. Any new gene target associated with such complex pathways involved in GBM tumorigenesis and evolution can lead to a far better understanding of the base mechanisms involved. Our perspective is that AVIL is currently a strong option to move towards the betterment of the standard of care and the outcomes of patients who are inflicted with this horrible disease.

Funding: HL is supported by NCI R01CA24061.

Institutional Review Board Statement: Not applicable.

Informed Consent Statement: Not applicable.

Acknowledgments: Figures created with BioRender.com, accessed on 15 December 2021.

Conflicts of Interest: The authors declare no conflict of interest.

\section{References}

1. Wen, P.Y.; Kesari, S. Malignant gliomas in adults. N. Engl. J. Med. 2008, 359, 492-507. [CrossRef]

2. Wesseling, P.; Capper, D. WHO 2016 Classification of gliomas. Neuropathol. Appl. Neurobiol. 2018, 44, 139-150. [CrossRef]

3. Batich, K.A.; Sampson, J.H. Standard of care and future pharmacological treatment options for malignant glioma: An urgent need for screening and identification of novel tumor-specific antigens. Expert Opin. Pharmacother. 2014, 15, 2047-2061. [CrossRef]

4. Tan, A.C.; Ashley, D.M.; López, G.Y.; Malinzak, M.; Friedman, H.S.; Khasraw, M. Management of glioblastoma: State of the art and future directions. CA Cancer J. Clin. 2020, 70, 299-312. [CrossRef]

5. Huang, B.; Li, X.; Li, Y.; Zhang, J.; Zong, Z.; Zhang, H. Current Immunotherapies for Glioblastoma Multiforme. Front. Immunol. 2020, 11, 603911. [CrossRef] [PubMed]

6. Weller, M.; Cloughesy, T.; Perry, J.R.; Wick, W. Standards of care for treatment of recurrent glioblastoma-are we there yet? Neuro. Oncol. 2013, 15, 4-27. [CrossRef]

7. Johnson, D.R.; O'Neill, B.P. Glioblastoma survival in the United States before and during the temozolomide era. J. Neurooncol. 2012, 107, 359-364. [CrossRef] [PubMed]

8. Ohgaki, H.; Dessen, P.; Jourde, B.; Horstmann, S.; Nishikawa, T.; Di Patre, P.-L.; Burkhard, C.; Schüler, D.; Probst-Hensch, N.M.; Maiorka, P.C.; et al. Genetic pathways to glioblastoma: A population-based study. Cancer Res. 2004, 64, 6892-6899. [CrossRef]

9. Kleihues, P.; Ohgaki, H. Primary and secondary glioblastomas: From concept to clinical diagnosis. Neuro. Oncol. 1999, 1, 44-51. [CrossRef]

10. Das, P.; Puri, T.; Jha, P.; Pathak, P.; Joshi, N.; Suri, V.; Sharma, M.C.; Sharma, B.S.; Mahapatra, A.K.; Suri, A.; et al. A clinicopathological and molecular analysis of glioblastoma multiforme with long-term survival. J. Clin. Neurosci. 2011, 18, 66-70. [CrossRef] [PubMed]

11. Krex, D.; Klink, B.; Hartmann, C.; von Deimling, A.; Pietsch, T.; Simon, M.; Sabel, M.; Steinbach, J.P.; Heese, O.; Reifenberger, G.; et al. German Glioma Network Long-term survival with glioblastoma multiforme. Brain 2007, 130, 2596-2606. [CrossRef] [PubMed]

12. Hambardzumyan, D.; Bergers, G. Glioblastoma: Defining Tumor Niches. Trends Cancer 2015, 1, 252. [CrossRef] [PubMed]

13. Brat, D.J.; Van Meir, E.G. Vaso-occlusive and prothrombotic mechanisms associated with tumor hypoxia, necrosis, and accelerated growth in glioblastoma. Lab. Investig. 2004, 84, 397-405. [CrossRef]

14. Turcan, S.; Rohle, D.; Goenka, A.; Walsh, L.A.; Fang, F.; Yilmaz, E.; Campos, C.; Fabius, A.W.M.; Lu, C.; Ward, P.S.; et al. IDH1 mutation is sufficient to establish the glioma hypermethylator phenotype. Nature 2012, 483, 479-483. [CrossRef]

15. Han, S.; Liu, Y.; Cai, S.J.; Qian, M.; Ding, J.; Larion, M.; Gilbert, M.R.; Yang, C. IDH mutation in glioma: Molecular mechanisms and potential therapeutic targets. Br. J. Cancer 2020, 122, 1580-1589. [CrossRef]

16. Zhang, P.; Xia, Q.; Liu, L.; Li, S.; Dong, L. Current Opinion on Molecular Characterization for GBM Classification in Guiding Clinical Diagnosis, Prognosis, and Therapy. Front. Mol. Biosci. 2020, 7, 241. [CrossRef]

17. Komori, T. Grading of adult diffuse gliomas according to the 2021 WHO Classification of Tumors of the Central Nervous System. Lab. Investig. 2021, 23, 1231-1251. [CrossRef] [PubMed] 
18. Louis, D.N.; Perry, A.; Wesseling, P.; Brat, D.J.; Cree, I.A.; Figarella-Branger, D.; Hawkins, C.; Ng, H.K.; Pfister, S.M.; Reifenberger, G.; et al. The 2021 WHO Classification of Tumors of the Central Nervous System: A summary. Neuro. Oncol. 2021, 23, 1231-1251. [CrossRef] [PubMed]

19. Weller, M.; Le Rhun, E. How did lomustine become standard of care in recurrent glioblastoma? Cancer Treat. Rev. 2020, 87, 102029. [CrossRef] [PubMed]

20. Wick, W.; Gorlia, T.; Bendszus, M.; Taphoorn, M.; Sahm, F.; Harting, I.; Brandes, A.A.; Taal, W.; Domont, J.; Idbaih, A.; et al. Lomustine and Bevacizumab in Progressive Glioblastoma. N. Engl. J. Med. 2017, 377, 1954-1963. [CrossRef]

21. Ren, X.; Ai, D.; Li, T.; Xia, L.; Sun, L. Effectiveness of Lomustine Combined With Bevacizumab in Glioblastoma: A Meta-Analysis. Front. Neurol. 2021, 11, 1893. [CrossRef] [PubMed]

22. Agarwal, S.; Sane, R.; Oberoi, R.; Ohlfest, J.R.; Elmquist, W. Delivery of Molecularly Targeted Therapy to Malignant Glioma, a Disease of the Whole Brain. Expert Rev. Mol. Med. 2011, 13, e17. [CrossRef]

23. Lathia, J.D.; Mack, S.C.; Mulkearns-Hubert, E.E.; Valentim, C.L.L.; Rich, J.N. Cancer stem cells in glioblastoma. Genes Dev. 2015, 29, 1203. [CrossRef] [PubMed]

24. Liebelt, B.D.; Shingu, T.; Zhou, X.; Ren, J.; Shin, S.A.; Hu, J. Glioma Stem Cells: Signaling, Microenvironment, and Therapy. Stem Cells Int. 2016, 2016, 7849890. [CrossRef]

25. Livshits, Z.; Rao, R.B.; Smith, S.W. An approach to chemotherapy-associated toxicity. Emerg. Med. Clin. North Am. 2014, 32, 167-203. [CrossRef] [PubMed]

26. Druker, B.J.; Sawyers, C.L.; Kantarjian, H.; Resta, D.J.; Reese, S.F.; Ford, J.M.; Capdeville, R.; Talpaz, M. Activity of a specific inhibitor of the BCR-ABL tyrosine kinase in the blast crisis of chronic myeloid leukemia and acute lymphoblastic leukemia with the Philadelphia chromosome. N. Engl. J. Med. 2001, 344, 1038-1042. [CrossRef] [PubMed]

27. Ascierto, P.A.; McArthur, G.A.; Dréno, B.; Atkinson, V.; Liszkay, G.; Di Giacomo, A.M.; Mandalà, M.; Demidov, L.; Stroyakovskiy, D.; Thomas, L.; et al. Cobimetinib combined with vemurafenib in advanced BRAF(V600)-mutant melanoma (coBRIM): Updated efficacy results from a randomised, double-blind, phase 3 trial. Lancet. Oncol. 2016, 17, 1248-1260. [CrossRef]

28. Robert, C.; Karaszewska, B.; Schachter, J.; Rutkowski, P.; Mackiewicz, A.; Stroiakovski, D.; Lichinitser, M.; Dummer, R.; Grange, F.; Mortier, L.; et al. Improved overall survival in melanoma with combined dabrafenib and trametinib. N. Engl. J. Med. 2015, 372, 30-39. [CrossRef]

29. Greco, A.; Safi, D.; Swami, U.; Ginader, T.; Milhem, M.; Zakharia, Y. Efficacy and Adverse Events in Metastatic Melanoma Patients Treated with Combination BRAF Plus MEK Inhibitors Versus BRAF Inhibitors: A Systematic Review. Cancers (Basel) 2019, 11, 1950. [CrossRef] [PubMed]

30. Dummer, R.; Ascierto, P.A.; Gogas, H.J.; Arance, A.; Mandala, M.; Liszkay, G.; Garbe, C.; Schadendorf, D.; Krajsova, I.; Gutzmer, R.; et al. Encorafenib plus binimetinib versus vemurafenib or encorafenib in patients with BRAF-mutant melanoma (COLUMBUS): A multicentre, open-label, randomised phase 3 trial. Lancet. Oncol. 2018, 19, 603-615. [CrossRef]

31. Perez, E.A. Microtubule inhibitors: Differentiating tubulin-inhibiting agents based on mechanisms of action, clinical activity, and resistance. Mol. Cancer Ther. 2009, 8, 2086-2095. [CrossRef]

32. Diaz-Padilla, I.; Oza, A.M. Epothilones in the treatment of ovarian cancer. Futur. Oncol. 2011, 7, 559-568. [CrossRef] [PubMed]

33. Zagouri, F.; Sergentanis, T.N.; Chrysikos, D.; Dimopoulos, M.A.; Bamias, A. Epothilones in epithelial ovarian, fallopian tube, or primary peritoneal cancer: A systematic review. Onco. Targets. Ther. 2015, 8, 2187-2198. [CrossRef] [PubMed]

34. Xie, Z.; Janczyk, P.Ł.; Zhang, Y.; Liu, A.; Shi, X.; Singh, S.; Facemire, L.; Kubow, K.; Li, Z.; Jia, Y.; et al. A cytoskeleton regulator AVIL drives tumorigenesis in glioblastoma. Nat. Commun. 2020, 11, 3457. [CrossRef]

35. Marks, P.W.; Arai, M.; Bandura, J.L.; Kwiatkowski, D.J. Advillin (p92): A new member of the gelsolin/villin family of actin regulatory proteins. J. Cell Sci. 1998, 111. [CrossRef]

36. Silacci, P.; Mazzolai, L.; Gauci, C.; Stergiopulos, N.; Yin, H.L.; Hayoz, D. Gelsolin superfamily proteins: Key regulators of cellular functions. Cell. Mol. Life Sci. 2004, 61, 2614-2623. [CrossRef]

37. Nag, S.; Larsson, M.; Robinson, R.C.; Burtnick, L.D. Gelsolin: The tail of a molecular gymnast. Cytoskeleton 2013, 70, 360-384. [CrossRef]

38. Izdebska, M.; Zielińska, W.; Grzanka, D.; Gagat, M. The Role of Actin Dynamics and Actin-Binding Proteins Expression in Epithelial-to-Mesenchymal Transition and Its Association with Cancer Progression and Evaluation of Possible Therapeutic Targets. Biomed Res. Int. 2018, 2018, 4578373. [CrossRef] [PubMed]

39. Rao, J.; Ashraf, S.; Tan, W.; Van Der Ven, A.T.; Gee, H.Y.; Braun, D.A.; Fehér, K.; George, S.P.; Esmaeilniakooshkghazi, A.; Choi, W.; et al. Advillin acts upstream of phospholipase C $€ 1$ in steroid-resistant nephrotic syndrome. J. Clin. Invest. 2017, 127, 4257-4269. [CrossRef]

40. George, S.P.; Esmaeilniakooshkghazi, A.; Roy, S.; Khurana, S. F-actin-bundling sites are conserved in proteins with villin-type headpiece domains. Mol. Biol. Cell 2020, 31, 1857-1866. [CrossRef]

41. Vermeulen, W.; Vanhaesebrouck, P.; Van Troys, M.; Verschueren, M.; Fant, F.; Goethals, M.; Ampe, C.; Martins, J.C.; Borremans, F.A.M. Solution structures of the C-terminal headpiece subdomains of human villin and advillin, evaluation of headpiece F-actin-binding requirements. Protein Sci. 2004, 13, 1276-1287. [CrossRef] [PubMed]

42. Matarrese, P.; Vona, R.; Ascione, B.; Paggi, M.G.; Mileo, A.M. Physical Interaction between HPV16E7 and the Actin-Binding Protein Gelsolin Regulates Epithelial-Mesenchymal Transition via HIPPO-YAP Axis. Cancers (Basel) 2021, 13, 353. [CrossRef] 
43. Oelrich, F.; Junker, H.; Stope, M.; Erb, H.H.; Walther, R.; Venz, S.; Zimmermann, U. Gelsolin governs the neuroendocrine transdifferentiation of prostate cancer cells and suppresses the apoptotic machinery. Anticancer Res. 2021, 41, 3717-3729. [CrossRef]

44. Miura, N.; Takemori, N.; Kikugawa, T.; Tanji, N.; Higashiyama, S.; Yokoyama, M. Adseverin: A novel cisplatin-resistant marker in the human bladder cancer cell line HT1376 identified by quantitative proteomic analysis. Mol. Oncol. 2012, 6, 311-322. [CrossRef]

45. Lin, Q.; Li, J.; Zhu, D.; Niu, Z.; Pan, X.; Xu, P.; Ji, M.; Wei, Y.; Xu, J. Aberrant Scinderin Expression Correlates With Liver Metastasis and Poor Prognosis in Colorectal Cancer. Front. Pharmacol. 2019, 10, 1183. [CrossRef]

46. Maklad, A.; Sharma, A.; Azimi, I. Calcium Signaling in Brain Cancers: Roles and Therapeutic Targeting. Cancers (Basel) 2019, 11, 145. [CrossRef]

47. Strudwick, X.L.; Cowin, A.J. Multifunctional Roles of the Actin-Binding Protein Flightless I in Inflammation, Cancer and Wound Healing. Front. Cell Dev. Biol. 2020, 8, 1394. [CrossRef] [PubMed]

48. Gdynia, G.; Grund, K.; Eckert, A.; Böck, B.C.; Funke, B.; Macher-Goeppinger, S.; Sieber, S.; Herold-Mende, C.; Wiestler, B.; Wiestler, O.D.; et al. Basal caspase activity promotes migration and invasiveness in glioblastoma cells. Mol. Cancer Res. 2007, 5, 1232-1240. [CrossRef]

49. Qiao, X.; Zhou, Y.; Xie, W.; Wang, Y.; Zhang, Y.; Tian, T.; Dou, J.; Yang, X.; Shen, S.; Hu, J.; et al. Scinderin is a novel transcriptional target of BRMS1 involved in regulation of hepatocellular carcinoma cell apoptosis. Am. J. Cancer Res. 2018, 8, 1008-1018. [PubMed]

50. Asare-Werehene, M.; Communal, L.; Carmona, E.; Han, Y.; Song, Y.S.; Burger, D.; Mes-Masson, A.M.; Tsang, B.K. Plasma gelsolin inhibits CD8p t-cell function and regulates glutathione production to confer chemoresistance in ovarian cancer. Cancer Res. 2020, 80, 3959-3971. [CrossRef] [PubMed]

51. Asare-Werehene, M.; Nakka, K.; Reunov, A.; Chiu, C.T.; Lee, W.T.; Abedini, M.R.; Wang, P.W.; Shieh, D.-B.; Dilworth, F.J.; Carmona, E.; et al. The exosome-mediated autocrine and paracrine actions of plasma gelsolin in ovarian cancer chemoresistance. Oncogene 2020, 39, 1600-1616. [CrossRef]

52. Chiu, C.T.; Wang, P.W.; Asare-Werehene, M.; Tsang, B.K.; Shieh, D. Bin Circulating plasma gelsolin: A predictor of favorable clinical outcomes in head and neck cancer and sensitive biomarker for early disease diagnosis combined with soluble fas ligand Cancers (Basel) 2020, 12, 1569. [CrossRef]

53. Prescher, N.; Hänsch, S.; Knobbe-Thomsen, C.B.; Stühler, K.; Poschmann, G. The migration behavior of human glioblastoma cells is influenced by the redox-sensitive human macrophage capping protein CAPG. Free Radic. Biol. Med. 2021, 167, 81-93. [CrossRef] [PubMed]

54. Lang, Z.; Chen, Y.; Zhu, H.; Sun, Y.; Zhang, H.; Huang, J.; Zou, Z. Prognostic and clinicopathological significance of CapG in various cancers: Evidence from a meta-analysis. Pathol. Res. Pract. 2019, 215, 152683. [CrossRef] [PubMed]

55. Yun, D.-P.; Wang, Y.-Q.; Meng, D.-L.; Ji, Y.-Y.; Chen, J.-X.; Chen, H.-Y.; Lu, D.-R. Actin-capping protein CapG is associated with prognosis, proliferation and metastasis in human glioma. Oncol. Rep. 2018, 39, 1011-1022. [CrossRef] [PubMed]

56. Huang, S.; Chi, Y.; Qin, Y.; Wang, Z.; Xiu, B.; Su, Y.; Guo, R.; Guo, L.; Sun, H.; Zeng, C.; et al. CAPG enhances breast cancer metastasis by competing with PRMT5 to modulate STC-1 transcription. Theranostics 2018, 8, 2549-2564. [CrossRef]

57. Koya, R.C.; Fujita, H.; Shimizu, S.; Ohtsu, M.; Takimoto, M.; Tsujimoto, Y.; Kuzumaki, N. Gelsolin Inhibits Apoptosis by Blocking Mitochondrial Membrane Potential Loss and Cytochrome c Release. J. Biol. Chem. 2000, 275, 15343-15349. [CrossRef]

58. Wang, Z.; Zhang, S.; Siu, T.L.; Huang, S. Glioblastoma Multiforme Formation and EMT: Role of FoxM1 Transcription Factor. Curr. Pharm. Des. 2015, 21, 1268. [CrossRef] [PubMed]

59. Myatt, S.S.; Lam, E.W.-F. The emerging roles of forkhead box (Fox) proteins in cancer. Nat. Rev. Cancer 2007, 7, 847-859. [CrossRef] [PubMed]

60. Liu, M.; Dai, B.; Kang, S.-H.; Ban, K.; Huang, F.-J.; Lang, F.F.; Aldape, K.D.; Xie, T.; Pelloski, C.E.; Xie, K.; et al. FoxM1B Is Overexpressed in Human Glioblastomas and Critically Regulates the Tumorigenicity of Glioma Cells. Cancer Res. 2006, 66, 3593-3602. [CrossRef] [PubMed]

61. Qin, R.; Zhou, J.; Chen, C.; Xu, T.; Yan, Y.; Ma, Y.; Zheng, Z.; Shen, Y.; Lu, Y.; Fu, D.; et al. LIN28 is involved in glioma carcinogenesis and predicts outcomes of glioblastoma multiforme patients. PLoS ONE 2014, 9, e86446. [CrossRef]

62. Viswanathan, S.R.; Powers, J.T.; Einhorn, W.; Hoshida, Y.; Ng, T.L.; Toffanin, S.; O'Sullivan, M.; Lu, J.; Phillips, L.A.; Lockhart, V.L.; et al. Lin28 promotes transformation and is associated with advanced human malignancies. Nat. Genet. 2009, 41, 843-848. [CrossRef]

63. Mao, X.; Hütt-Cabezas, M.; Orr, B.A.; Weingart, M.; Taylor, I.; Rajan, A.K.D.; Odia, Y.; Kahlert, U.; Maciaczyk, J.; Nikkhah, G.; et al. LIN28A facilitates the transformation of human neural stem cells and promotes glioblastoma tumorigenesis through a pro-invasive genetic program. Oncotarget 2013, 4, 1050. [CrossRef] [PubMed]

64. Jiang, Y.; Li, W.; Yan, Y.; Yao, X.; Gu, W.; Zhang, H. LINC01094 triggers radio-resistance in clear cell renal cell carcinoma via miR-577/CHEK2/FOXM1 axis. Cancer Cell Int. 2020, 20, 274. [CrossRef]

65. Luo, C.; Lin, K.; Hu, C.; Zhu, X.; Zhu, J.; Zhu, Z. LINC01094 promotes pancreatic cancer progression by sponging miR-577 to regulate LIN28B expression and the PI3K/AKT pathway. Mol. Ther.-Nucleic Acids 2021, 26, 523-535. [CrossRef] [PubMed]

66. Yilmaz, M.; Christofori, G. EMT, the cytoskeleton, and cancer cell invasion. Cancer Metastasis Rev. 2009, 28, 15-33. [CrossRef]

67. Hall, A. The cytoskeleton and cancer. Cancer Metastasis Rev. 2009, 28, 5-14. [CrossRef] 
68. Klymkowsky, M.W.; Savagner, P. Epithelial-mesenchymal transition: A cancer researcher's conceptual friend and foe. Am. J. Pathol. 2009, 174, 1588-1593. [CrossRef] [PubMed]

69. Smyth, M.J.; Hayakawa, Y.; Takeda, K.; Yagita, H. New aspects of natural-killer-cell surveillance and therapy of cancer. Nat. Rev. Cancer 2002, 2, 850-861. [CrossRef] [PubMed]

70. Al Absi, A.; Wurzer, H.; Guerin, C.; Hoffmann, C.; Moreau, F.; Mao, X.; Brown-Clay, J.; Petrolli, R.; Casellas, C.P.; Dieterle, M.; et al. Actin Cytoskeleton Remodeling Drives Breast Cancer Cell Escape from Natural Killer-Mediated Cytotoxicity. Cancer Res. 2018, 78, 5631-5643. [CrossRef]

71. Iser, I.C.; Pereira, M.B.; Lenz, G.; Wink, M.R. The Epithelial-to-Mesenchymal Transition-Like Process in Glioblastoma: An Updated Systematic Review and In Silico Investigation. Med. Res. Rev. 2017, 37, 271-313. [CrossRef] [PubMed] 